WORK-RELATED FACTORS THAT INFLUENCE RETIREMENT DECISIONS IN THE UK: THE HEALTH AND EMPLOYMENT AFTER FIFTY FACTORS INFLUENCING RETIREMENT STUDY (HEAF FIRST)

\footnotetext{
1,2Martin Stevens*, 'Mary Barker, 1,2Stefania D'Angelo, 'Elaine Dennison, ${ }^{1,2}$ E Clare Harris, ${ }^{1,2}$ Cathy Linaker, ${ }^{1,2}$ Holly Syddall, ${ }^{1,2}$ Karen Walker-Bone. ${ }^{1} M R C$ Lifecourse Epidemiology Unit, University of Southampton, Southampton, UK; ${ }^{2}$ Versus Arthritis/MRC Centre for Musculoskeletal Health and Work, University of Southampton, Southampton, UK
}

\subsection{6/jech-2021-SSMabstracts.27}

Background Population demographics are changing due to increased longevity and declining birth rates, precipitating a demand to encourage people to work to older ages. Whilst legislative changes have enabled this, little is currently known about factors which employers could influence within workplaces to encourage later working. We investigated the role of work-related factors in retirement decisions in the UK, utilising the Health and Employment After Fifty (HEAF) prospective cohort study.

Methods The HEAF cohort was incepted in 2013-14 using the sampling frame of 24 GP surgeries across England. People aged 50-64 years at baseline completed annual questionnaires about health, work and retirement plans. HEAF FIRST involves mixed-methods research starting with qualitative interviews and a systematic review preceding and informing the design of a nested case-control study. A questionnaire was designed to investigate the role of workrelated factors on retirement status. Questionnaires were sent to people who were employed at baseline but selfreported retirement by 2018 (cases) and people who were working at baseline and remained employed in 2018, matched on age +/- 2 years and sex. Those who retired mainly for health reasons were excluded. The relationship between work-related factors and retirement was investigated using logistic regression models adjusted for age and sex (matching factors), finances, marital, and socio-economic status.

Results Questionnaires were posted to 570 cases and 570 controls. In total, responses were received from 488 retirees and 448 current workers. On average, retirees (61\% women) were one-year older, financially better off and of higher socio-economic position than workers $(62 \%$ women $)$. Retirees had retired at mean age 61.6 years, $64 \%$ pre-State Pension Age (SPA), $11 \%$ at SPA (+/-6 months) and $25 \%$ post-SPA. The following work factors were associated with increased odds of retirement (all results adjusted for factors detailed above): effort reward imbalance, (OR 1.43, 95\%CI 1.26,1.63), less flexibility (OR 1.25, 95\%CI 1.10,1.42), constant availability (OR 1.30 95\%CI 1.14,1.49) work-life conflict (OR $1.3595 \%$ CI 1.18,1.55) high demand/low control (OR 2.00, 95\%CI $1.33,3.01)$.

Discussion Our results suggest that work-related factors play an important role in retirement. People who are working in jobs which offer flexibility, are rewarding, minimise conflict with home life and offer autonomy are more likely to report working to older ages. The work-related factors identified can be utilised to inform employer-based interventions to encourage people to work to older ages.

\section{OP28 THE IMPACT OF HIGH-RISK MEDICATIONS ON MORTALITY RISK AMONG OLDER ADULTS WITH POLYPHARMACY IN ENGLAND}

${ }^{1}$ Yun-Ting Huang ${ }^{*},{ }^{2}$ Andrew Steptoe, ${ }^{3}$ Li Wei, ${ }^{1}$ Paola Zaninotto. ${ }^{1}$ Epidemiology and Public Health, University College London, London, UK; ${ }^{2}$ Behavioural Science and Health, University College London, London, UK; ${ }^{3}$ School of Pharmacy, University College London, London, UK

\subsection{6/jech-2021-SSMabstracts.28}

Background Polypharmacy is common among older people and found to be associated with a high risk of mortality. However, little is known about how specific medications impact older adults with polypharmacy. Thus, this study aimed to investigate associations between high-risk medications and all-cause and cause-specific mortality among older adults with polypharmacy.

Methods Data came from Wave 6 (2012/2013) of the English Longitudinal Study of Ageing, a nationally representative sample of people aged 50 and older. 1705 participants with polypharmacy, defined as taking five or more long-term medications a day for conditions or symptoms, were included. First, using agglomerative hierarchical clustering method participants were grouped according to the use of 14 high-risk medication categories. Five clusters of high-risk medications were identified. Next, the relationship between the five highrisk medications clusters and all-cause and cause-specific mortality (followed up to March 2018) was examined. All-cause mortality was assessed by Cox proportional hazards model and competing-risks regression was employed for cause-specific mortality.

Results Five high-risk medication clusters were identified: 1) RAAS inhibitors 2) mental drugs 3) CNS drugs 4) RAAS inhibitors and antithrombotics and 5) antithrombotics. The mental drugs cluster showed higher risks of all-cause $(\mathrm{HR}=1.55,95 \% \mathrm{CI}=1.05,2.28)$ and $\mathrm{CVD}(\mathrm{SHR}=2.11,95 \%$ $\mathrm{CI}=1.10$, 4.05) mortality than CNS drugs cluster over six years, while others showed no differences in mortality. The mental drugs cluster showed the highest prevalence of antidepressants (64.1\%), benzodiazepines (10.4\%), antipsychotics (2.4\%), antimanics (0.7\%), opioids (33.2\%) and muscle relaxants $(21.5 \%)$ amongst the five medication patterns. The findings suggest that older adults with polypharmacy who take mental drugs (primarily antidepressants), opioids and muscle relaxants are prone to increase the risk of all-cause and CVD mortality, compared with those who did not take these types of medications.

*Abbreviation: RAAS: renin-angiotensin-aldosterone system, CNS: central nervous system, CVD: cardiovascular disease.

Conclusion The use of mental drugs, opioids, and muscle relaxants was found to add on mortality risk to communitydwelling older people with polypharmacy in England. This study supports the view that addictive pain management medications should be included in structured medication reviews, but also suggests that the prescription of mental medications and muscle relaxants in older adults with polypharmacy may need more attention. The reinforcement of structured medication reviews would contribute to the early intervention in medication use and may help reduce the mortality risk of people with polypharmacy. 